\title{
Effects of external environment on thermocapillary convection of high prandtl number fluid
}

\author{
Ruquan Liang ${ }^{1}$,Fuqiang Yan ${ }^{1}$, Xiaoyuan $\mathrm{Li}^{1}$, Shuo Zhang ${ }^{1}$ and Shuo Yang ${ }^{1}$ \\ ${ }^{1}$ Key Laboratory of National Education Ministry for Electromagnetic Processes of Materials, Northeastern University in Shen Yang, CN.
}

\begin{abstract}
Numerical simulations have been carried out to investigate the influence of external environment on thermocapillary convection in high Prandtl number $(P r=68)$ liquid. The geometric model of physical problem is that the the liquid bridge surrounded by ambient air under zero or ground gravity. The interface velocity, temperature, heat flux and flow pattern in the liquid bridge are presented and discussed under different conditions by changing the external environment. The buoyancy convection produces a symmetrical vortex in the liquid bridge. The ambient air affects the distributions of the temperature velocity and heat flux on the interface by changing the thermocapillary convection.
\end{abstract}

\section{Introduction}

In present industrial practice, the floating zone method is used for growing the high quality crystal. Under this background, the liquid bridge is a physical model, which is often used to simulate industrial floating half-zone crystal growth technology ${ }^{[1]}$.

To research the effect of external environment on liquid bridge, Kamotani. $\mathrm{Y}$ et al. ${ }^{[2]}$ put the liquid bridge into incubator and changed its temperature, and their results illustrated that critical parameters strongly depend on temperature of ambient air. In another experiment ${ }^{[3]}$, a thin plastic plate was placed in the vicinity of liquid bridge did not contact with the interface. The changes in temperature and position of plastic plate affected the heat transfer between plastic plate and liquid bridge interface, which caused the change in the stability of liquid bridge interface. Mialdum. A et al. ${ }^{[4-5]}$ carried out the research about the liquid bridge with deflector shield. They illustrated that the critical temperature and oscillation frequency strongly depend on the ambient conditions and temperature of deflector shield. Shevtsova. $\mathrm{V}$ et al ${ }^{[6]}$ found that the heat absorption weakens the flow of interface, and the heat liberation strengthens the flow. In addition, Shevtsova. $\mathrm{V}^{[7]}$ carried out the experiment about the flow pattern in the liquid bridge, which illustrated that the buoyancy convection produces a vortex in the liquid bridge under normal gravity. However, almost experiments were carried out under normal gravity, there is still contention on the effect of the air with different temperature and velocity on thermocapillary flow under zero gravity.

\section{Physical model}

The physical model is shown in Figure 1. The $5 \mathrm{cSt}$ silicone is selected as the liquid, and the air is selected as the ambient air. The system consists of one cylinder and two solid rods. The cylinder is the glass tube with radius of $\mathrm{R}_{\text {out }}$, and the two solid rods are the upper and lower rod with radius of radius of $\mathrm{R}_{0}$, respectively. The liquid bridge is injected between the two solid rods. 


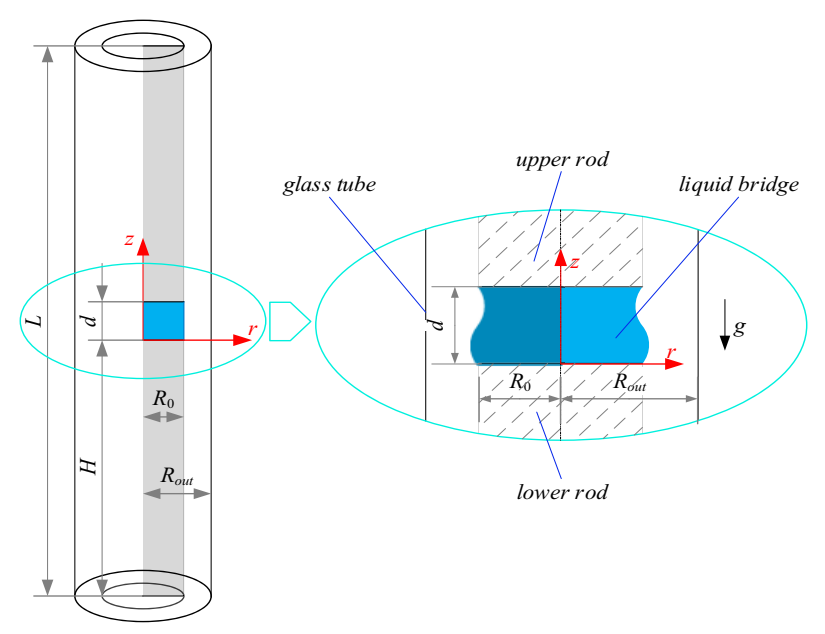

Figure 1.Semi-floating zone liquid bridge model

The length of the glass tube is long enough to ensure the full development of the airflow. The specific geometric scales are shown in Table 1.

Table 1. Geometrical scales used in the calculations

\begin{tabular}{ccccc}
\hline $\begin{array}{c}R_{\text {out }} \\
(\mathrm{mm})\end{array}$ & $\begin{array}{c}R_{0} \\
(\mathrm{~mm})\end{array}$ & $\begin{array}{c}H \\
(\mathrm{~mm})\end{array}$ & $\begin{array}{c}d \\
(\mathrm{~mm})\end{array}$ & $\begin{array}{c}L \\
(\mathrm{~mm})\end{array}$ \\
\hline 5 & 3 & 20 & 3 & 43 \\
\hline
\end{tabular}

\section{Controlling equations}

As shown in Figure 1, the system dynamics is described by the continuity equation, momentum conservation equation and energy conservation equation, where the fluid is assumed as incompressible Newtonian fluid, and the Boussinesq assumption is used.

$$
\begin{gathered}
\nabla \cdot \mathbf{U}=0 \quad(1) \\
\rho \frac{\partial \mathbf{U}}{\partial t}+\rho(\mathbf{U} \cdot \nabla) \mathbf{U}=-\nabla P+\rho v \nabla^{2} \mathbf{U}+\mathbf{F}_{S} \delta(r-R) \\
\frac{\partial T}{\partial t}+\mathbf{U} \cdot \nabla T-K \nabla^{2} T=0
\end{gathered}
$$

Where $\rho$ is the fluid density, $P$ is the fluidpressure, $T$ is the fluidtemperature, $K$ is the thermal diffusivity, $v$ is the kinematics viscosity coefficient.

The static shape of liquid bridge is obtained by solving the Young-Laplace equation. According to Young-Laplace equation, the relationship between the differential pressure on fluid interface and the surface tension can be expressed as

$$
p^{g}-p^{l}=p^{g}-\left(p_{0}^{l}-r^{l} g z\right)=s\left(\frac{1}{r_{1}}+\frac{1}{r_{2}}\right)
$$

Where $r_{1}$ and $r_{2}$ represent the principal radius of curvature of the curved interface in different directions, respectively.

In semi-floating zone liquid bridge surface tension flow, the surface tension acts only on the free surface of melt. The surface tension is expressed as: $\mathbf{F}_{S} \delta(r-R)$, where $\delta$ is the Kronecker operator, $\mathbf{F}_{S} \delta(r-R)$ represents the surface tension only acts on the free surface $(r=R), r$ can be expressed as:

$$
r=\sqrt{x^{2}+y^{2}}
$$

$\mathbf{F}_{S}$ can be shown as:

$$
\mathbf{F}_{S}=\frac{\partial \sigma}{\partial x} \mathbf{e}_{x}+\frac{\partial \sigma}{\partial y} \mathbf{e}_{y}+\frac{\partial \sigma}{\partial z} \mathbf{e}_{z}
$$

Where $\mathbf{e}_{x}, \mathbf{e}_{y}$ and $\mathbf{e}_{z}$ represent the velocity components in the $x, y$, and $z$ directions, respectively. $u, v$ and $w$ represent the unit vectors in $x, y$, and $z$, respectively.

Velocity boundary conditions:

The no-slip and no-flow-penetrating conditions are imposed on solid wall, its velocity components meet:

$$
u_{r}=u_{z}=0
$$

Temperature boundary conditions:

This study assumesthat the solid wall is isothermal, and the temperature of liquid in the vicinity of wallis equal to the temperature of wall:

$$
\begin{gathered}
T^{g}=T_{\text {amb }}=T_{0},\left(r=R_{\text {out }},-H<z<H+d\right) \\
T^{g}=T_{\text {cold }},\left(r=R_{o},-H<z<0\right) \\
T^{g}=T_{\text {hot }},\left(r=R_{o}, d<z<H+d\right) \\
T^{l}=T_{\text {cold }},\left(0<z<R_{0}, z=0\right) \\
T^{l}=T_{\text {hot }},\left(10<z<R_{0}, z=d\right)
\end{gathered}
$$

Where $T_{h o t}$ is the temperature of upper solid rod, $T_{\text {cold }}$ is the temperature of lower solid rod, $T_{a m b}$ is the ambient temperature,namely the temperature of outer cylinder, $T_{0}$ is the temperature of system average temperature,namely.

$$
T_{0}=\left(T_{\text {hot }}+T_{\text {cold }}\right) / 2
$$

\section{Results and discussions}


In gravity field, the shape of the liquid bridge changes greatly. The liquid bridge shape of the different volumes $\mathrm{V}$ and aspect ratiostare acquired by solving Young-Laplace equation using MATLAB software, and are shown in Figure2. In the present study, the liquid bridge of $\mathrm{V}=1$ and $\tau=1$ is analysed.

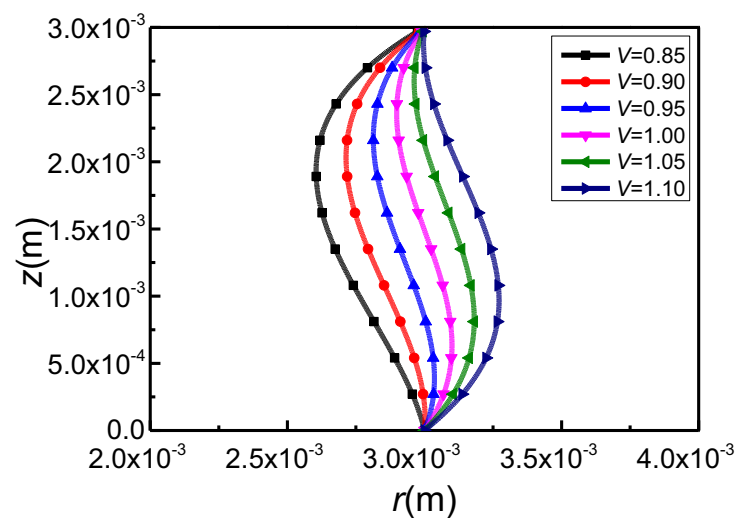

(a)

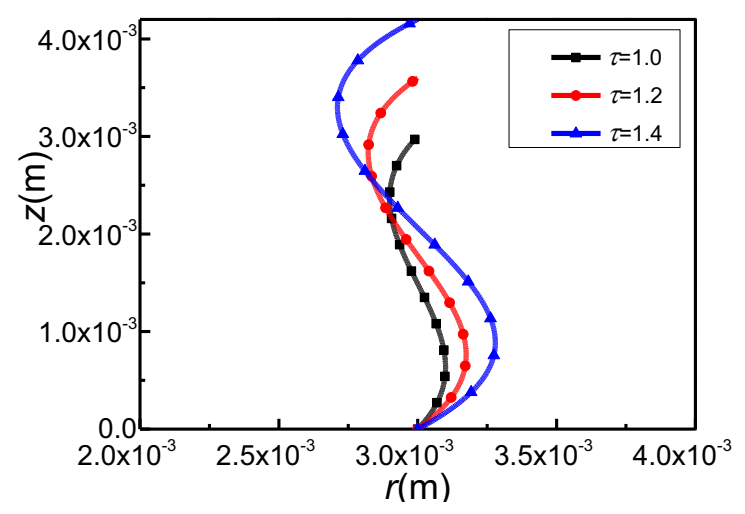

(b)

Figure2.(a) The equilibrium shapes of different volumesVin normal gravity when aspect ratio $\tau=1$.(b) The equilibrium shapes of different aspect ratios in normal gravity when volume $\mathrm{V}=1$.

Under the gravity field, the impact of buoyancy must be considered. As shown in the Figure 3 (a), the buoyancy convection produces a symmetrical vortex in the liquid bridge. Meanwhile, the shape of liquid bridge has changed under normal gravity, which is slightly concave in the upper and slightly convex in the lower. The convex value reaches the maximum at the $z=0.5$, and the concave value reaches the maximum at the $z=2.5$. The value of deformation is zero in the middle of liquid bridge, see Figure 3(b).

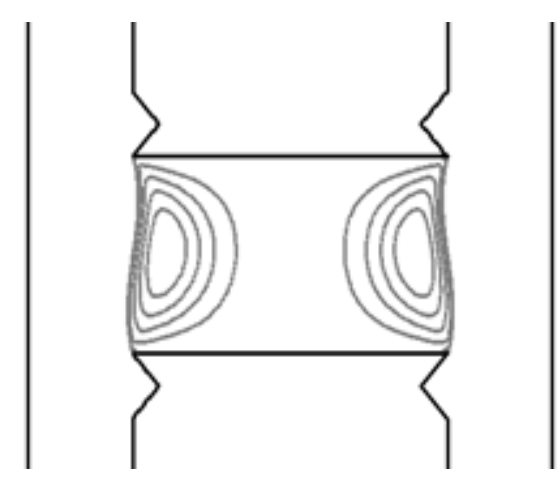

(a)

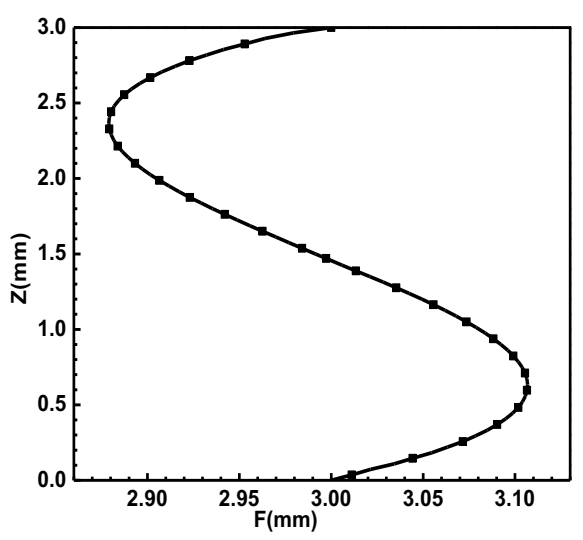

(b)

Figure3. Stream functions in the liquid bridge without forced gas motion, $P r=68, g=9.81 \mathrm{~m} / \mathrm{s}^{2}, \Delta T=5 \mathrm{~K}, u_{g}=0 \mathrm{~m} / \mathrm{s}, T_{a m b}=\mathrm{T}_{0}, \tau=1$ and $V=1$ (a) Flow pattern of the liquid bridge. (b) Interface of the liquid bridge

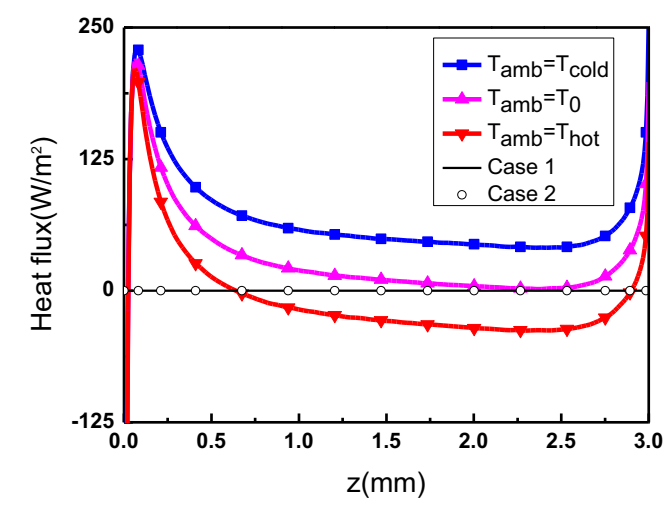

Figure4. Heat flux of the interface when $P r=68, g=0 \mathrm{~m} / \mathrm{s}^{2}$, $\Delta T=5 \mathrm{~K}, u_{\mathrm{g}}=0 \mathrm{~m} / \mathrm{s}, \tau=1$ and $V=1$. Case 1 corresponds to the case when the interface is adiabatic. Case 2 corresponds to the case when the influence of the ambient air is not considered

As shown in Figure 4, the heat flux of interface is negative at the cold end nearing wall, that is, the absorbing heat. This is because the temperature of fluid at the cold end is the lowest in the whole system. In addition, there is a heat flux peak at the hot end nearing wall, and its reason is similar to that of cold end, namely the temperature of 
fluid is the highest in the whole system. The heat flux of interface changes from positive value to negative one, that is, from heat absorption to heat liberation with the increase of ambient temperature. When the ambient temperature $T_{a m b}$ is equal to the temperature of cold end or average system, the liquid bridge is exothermic, and the liquid bridge is endothermic when the ambient temperature $T_{a m b}$ is equal to the hot end temperature $T_{h o t}$.

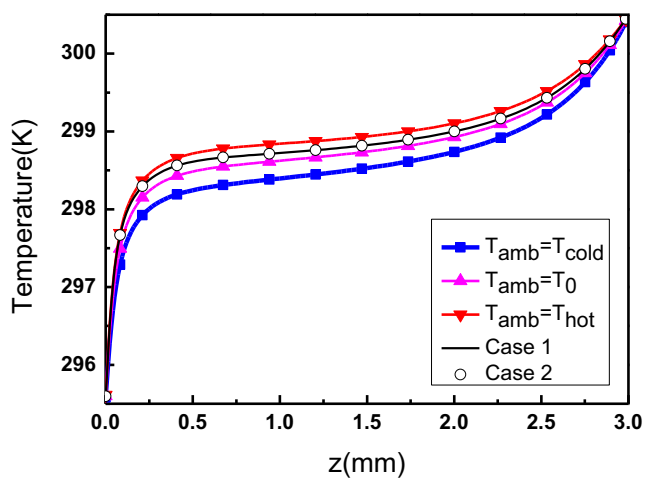

Figure 5.Temperature of the interface when $P r=68, g=0 \mathrm{~m} / \mathrm{s}^{2}$, $\Delta T=5 \mathrm{~K}, u_{g}=0 \mathrm{~m} / \mathrm{s}, \tau=1$ and $V=1$. Case 1 corresponds to the case when the interface is adiabatic. Case 2 corresponds to the case when the influence of the ambient air is not consider

As shown in the Figure 5, the temperature of liquid bridge interface reduces gradually from the hot end, and the changing range becomes more and more small. The temperature drops dramatically until nearing the cold end, and the temperature changes of both ends are more intense than that of the middle. The temperature change of cold end increases and the temperature change of hot end reduces. The interface temperature under adiabatic conditions is equal to the value between the average system temperature $T_{0}$ and hot end temperature $T_{h o t}$, which is close to the former in the vicinity of the cold end and is close to the latter in the vicinity of the hot end. The temperature distributions of the case 1 and case 2 are almost identical.

The flow in the interface is driven by the surface tension. The surface tension is changed by the ambient air, so the distribution of interface velocity is changed by the ambient air. As shown in Figure 6, there is a velocity peak at hot end and cold end, respectively. The velocity peak of cold end increases and the one of hot end reduces with the increase of ambient temperature, which corresponds to the Figure 5, namely the temperature gradient of cold end increases and the one of hot end decreases. The case 1 and 2 are almost identical.

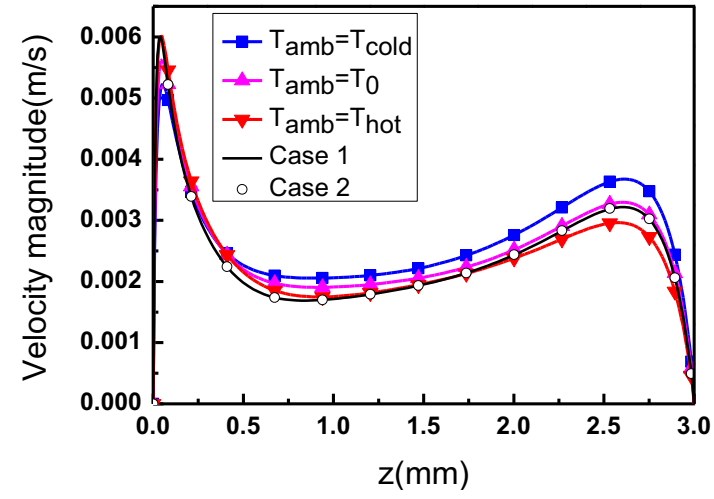

Figure6.Velocity of the interface when $P r=68$, $g=0 \mathrm{~m} / \mathrm{s}^{2}, \Delta T=5 \mathrm{~K}, u_{g}=0 \mathrm{~m} / \mathrm{s}, \tau=1$ and $V=1$. Case 1 corresponds to the case when the interface is adiabatic. Case 2 corresponds to the case when the influence of the ambient air is not considered

\section{5conclusions}

This paper establishes the geometry model of liquid bridge surrounded by the ambient air under zero or normal gravity, which is similar as the Kamotani'sexperiment ${ }^{[8]}$. Correspondingly, the mathematical model of interaction between the ambient air and liquid bridge is built. The parts of the boundary conditions are implemented by using the user-defined functions (User-Defined Function, UDF) and the Navier-Stokes equations of two-phase flow system are calculated by using the SIMPLEC algorithm. Finally, the numerical simulation of the effect of external environment on thermocapillary convection is completed. The conclusions are as follows:

1. The shape of liquid bridge changes under normal gravity, which is slightly concave in the upper and slightly convex in the lower. In addition, the buoyancy convection produces a symmetrical vortex in the liquid bridge.

2. Without the forced air, the effect of the air shear driven force is negligible compared with the effect of the ambient temperature.

3. When the liquid bridge is surrounded the air with the average system temperature, the temperature of interface is lower than that of no surrounding air. In addition, comparing with the adiabatic case, when the air temperature is equal to the temperature of hot end, the flow is suppressed; when the air temperature is equal to the cold end or system average temperature, the flow is strengthened, namely the heat absorption weakens flow, and the heat liberation strengthens the flow. 


\section{Acknowledgment}

The present work is supported financially by the National Natural Science Foundation of China under the grants of 51376040 and 11072057.

\section{Reference}

1. C.H. Chun, A micro-gravity simulation of the Marangoni convection. ActaAstronautica5(9), 681-686(1978)

2. Y. Kamotani, L. Wang, S. Hatta, Free surface heat loss effect on oscillatory thermocapillary flow in liquid bridges of high Prandtl number fluids. InternationalJournal of Heat and Mass Transfer 46(17),3211-3220 (2003)

3. A. Wang, Y. Kamotani, S. Yod, Oscillatory thermocapillary flow in liquid bridges of high Prandtl number fluid with free surface heat gain. InternationalJournal of Heat and Mass Transfer 50(21),4195-4205 (2007)

4. V. Shevtsova, A. Mialdun A, M. Mojahed, A study of heat transfer in liquid bridges near onset of instability. Journal of Non-equilibrium Thermodynamics 30(3), 261-281 (2005)

5. A. Mialdun, V. Shevtsova, Influence of interfacial heat exchange on the flow organization in liquid bridge. Microgravity-Science and Technology 18(3-4),146-149 (2006)

6. V.Shevtsova, Y. Gaponenko, A. Nepomnyashchy, Thermocapillary flow regimes and instability caused by a gas stream along the interface. Journal of Fluid Mechanics 714, 644-670 (2003)

7. V. Shevtsova, Thermal convection in liquid bridges with curved free surfaces: benchmark of numerical solutions. Journal of Crystal Growth 280(3), 632-651(2005)

8. A. Wang, Y. Kamotani, S. Yod, Oscillatory thermocapillary flow in liquid bridges of high Prandtl number fluid with free surface heat gain. International Journal of Heat and Mass Transfer 50(21), 4125-4160(2007) 